\title{
Radiation damage to multi-walled carbon nanotubes and their Raman vibrational modes
}

\author{
RAZIYEH KESHTMAND ${ }^{1, *}$, MOHAMAD-REZA KHANLARY ${ }^{1}$ and ZAHRA KESHTMAND ${ }^{2}$ \\ ${ }^{1}$ Physics Department, Faculty of Science, University of International Imam Khomeini, Qazvin, Iran \\ ${ }^{2}$ Faculty of Science and Research Branch, Islamic Azad University, Tehran, Iran
}

MS received 18 September 2014; accepted 17 August 2015

\begin{abstract}
Since their discovery in 1991 by Iijima, carbon nanotubes (CNTs) have been of great interest, both from a fundamental point of view and for future applications. As recent experimental and theoretical studies demonstrate, irradiation of CNTs with energetic particles can successfully be used for nano-engineering, e.g., for creating molecular junctions between the nanotubes, making nanotube-based quantum dots and composite materials with enhanced mechanical properties. In this work, the Raman spectroscopy investigation of nanotube defects created by irradiation of $\mathrm{Ar}^{+}$ions with various energies and doses was presented. In order to obtain more informative data, scanning electron microscope and thermal gravimetric analysis were carried out. Sheet resistivity of samples was also measured with the standard four-point probe technique. From the results, it was evident that the intensity ratio of the ' $D$ band' at around $1300 \mathrm{~cm}^{-1}$ to the intensity of the ' $G$ band' at $1590 \mathrm{~cm}^{-1}$, relative intensity of the $D$ to $G$ bands increases with enhancement of the irradiation dose. Comparing the intensity ratio $I_{\mathrm{D}^{\prime}} / I_{\mathrm{G}}$ (the $\mathrm{D}^{\prime}$ band was assigned to the lattice defects which occur inside the graphene atomic layer and induces a break in the two-dimensional translational symmetry) with $I_{\mathrm{D}} / I_{\mathrm{G}}$ tend to decide the $\mathrm{D}^{\prime}$ band treated as a defect. Electrical measurements showed that resistance of samples increases with enhancement of irradiation dose, clearly due to creation of more defects.
\end{abstract}

Keywords. Multiwall carbon nanotube; Raman; defects; irradiation.

\section{Introduction}

Carbon nanotubes (CNTs), ${ }^{1}$ have unique electronic and mechanical properties which can be used in many applications such as nanometre-scale electronic devices and reinforcement agents in composites. Multi-walled carbon nanotubes (MWCNTs) consist of several concentric cylindrical layers of rolled up graphene sheets they exhibit unique mechanical, electrical and thermal properties. ${ }^{2}$ The CNTs are regarded as perfect structure in most experimental work due to their high aspect ratio. Nevertheless, the structures having defects are preferred in some cases such as hydrogen storage, ${ }^{3}$ enhancement of chemical reaction via preferred sites, ${ }^{4}$ welding and crosslinking. ${ }^{2,3}$ When an energetic ion ${ }^{1}$ penetrates a solid, it collides with the nuclei and the electrons of the target. For the ion energies typically used in materials science (lower/much lower than several hundred MeVs), the slowing down of the ion can be separated into two different channels $^{2}$ electronic and nuclear stopping. These phenomena could occur either at the growth stage or later with postactions like ion beam irradiation and chemical treatment. ${ }^{3,5}$ Among them, ion irradiation is a well-controllable method to modify the structural defect morphology. The knocked-on atoms will be stabilized by losing their energy through multiple scattering in CNTs network until they are thermalized

\footnotetext{
*Author for correspondence (razi.keshtmand@yahoo.com)
}

and adsorbed on to the nanotube walls as adatoms. ${ }^{5}$ Raman spectroscopy is one of the most widely used techniques for structural defect identification in CNTs: the ratio of the intensity of the so-called 'D band' at around $1300 \mathrm{~cm}^{-1}$ to the intensity of the ' $\mathrm{G}$ band' at $1590 \mathrm{~cm}^{-1}$, in which one could qualitatively make a comparative study between intensity of the $\mathrm{G}$ and $\mathrm{D}$ bands. Skakalova et $a l^{6}$ reported the increase in the intensity of the D-mode and the dependence of normalized intensity $I_{\mathrm{D}} / I_{\mathrm{G}}$ on various low doses of $\mathrm{Ar}^{+}$. Osvath et $a l^{4}$ made a topographical study on the MWCNTs irradiated by $\mathrm{Ar}^{+}$ion in $30 \mathrm{keV}$ energy with low doses of bombardment. ${ }^{6}$ In this paper these investigations are carried out to make an almost complete study in both wide range of implantation energy and doses with Raman spectroscopy. Moreover, a detailed study of Ar-ion effects on the electrical conductivity is presented.

\section{Material and methods}

MWCNTs were provided from Plasma Chem GmbH Inc. with purity of more than 95\% MWCNTs, length of 1-10 $\mu \mathrm{m}$ with 3-15 walls. Regarding the importance of purity in this study, the as-prepared CNTs post-purified in two stages: (a) chemical elimination of dangling molecules and catalyst nano-particles in $\mathrm{HNO}_{3}: \mathrm{HCl}$ with $3: 1$ ratio for $2 \mathrm{~h}$ at room temperature (RT) and (b) thermal oxidation of amorphous carbon in $500^{\circ} \mathrm{C}$ for $1 \mathrm{~h}$. Thermal gravimetric 
analysis (TGA) was carried out with Rheumatic Scientific SDA 1500 apparatus to study the amounts of remained amorphous carbon and catalyst nano-particles. The samples were prepared by ultrasonification of $10 \mathrm{mg}$ CNTs in toluene for 60 min until a well-dispersed solution is obtained. Afterwards, the suspension was uniformly dispersed on an $\mathrm{Al}$ dish. After evaporation of toluene, samples were irradiated by $\mathrm{Ar}^{+}$ions. Their Raman spectra were measured using Jobin Yvon LabRam HR-800 Stokes mode at $532 \mathrm{~nm}$ frequencydoubled Nd: YAG laser excitation. The expected depth profiles of irradiated CNTs were estimated by TRIM simulation programme. In order to observe the modified structure of CNT walls, due to irradiation, scanning electron microscope (SEM) micrographs of irradiated samples are compared with that of as-prepared one. The sheet resistivity of thin MWCNTs film were carried out using the standard four-point probe analysis.

\section{Results}

\subsection{Material characterization}

Figure 1 shows the TGA curves of as-purified CNTs. The absence of significant weight loss before $550^{\circ} \mathrm{C}$ indicates that low amount of amorphous carbon is present after purification. Moreover, the weight loss of catalyst nano-particles ${ }^{7}$ after $700^{\circ} \mathrm{C}$ is attributed to catalyst nano-particles suggesting that very low amounts of metallic particles are loaded in the CNTs. According to these results, the high purity (more than $95 \%$ ) MWCNTs with diameter ranging from 5 to $15 \mathrm{~nm}$, are used as pristine samples.

\subsection{Raman study of irradiated CNTs}

Raman spectroscopy has been proved to be an extremely valuable tool to study nano-tube structure. A spectrum of a CNTs sample consists some main features: a strong peak at around $1300 \mathrm{~cm}^{-1}$, the so-called 'D line', assigned to both amorphous carbon and disordered graphitic material and a

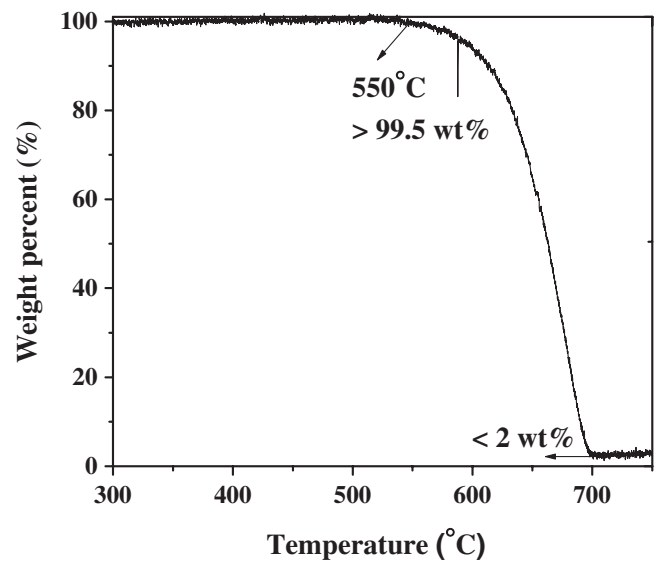

Figure 1. TGA thermogram of as-prepared CNTs. group of peaks in the approximate range of $1550-1600 \mathrm{~cm}^{-1}$. In particular a single peak at $1582 \mathrm{~cm}^{-1}$ labelled the ' $\mathrm{G}$ band' related to the tangential mode vibrations of the $\mathrm{C}$ atoms. The first-order Raman has another important and relatively weak disorder-induced band that appears as a shoulder next to the G peak. ${ }^{8}$ Raman spectra of pristine and irradiated with different doses are presented in figure 2. It is illustrated that the graphitic structure of CNTs is destroyed under ion beam irradiation and the full-width at half-maximum (FWHM) of the $\mathrm{D}$ band is increased with irradiation dose (figure 3 ). The

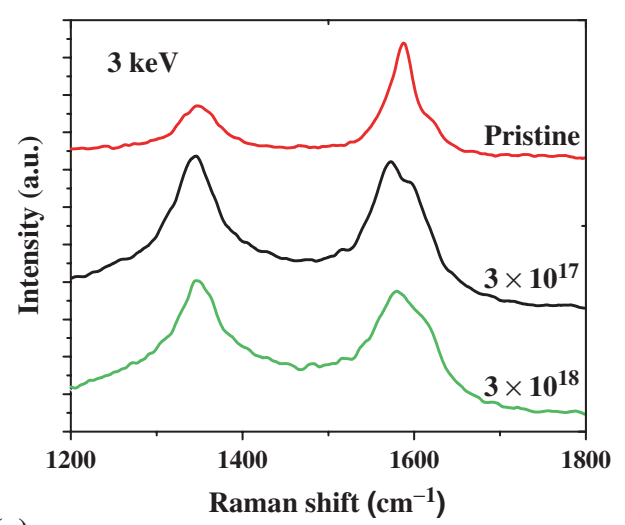

(a)

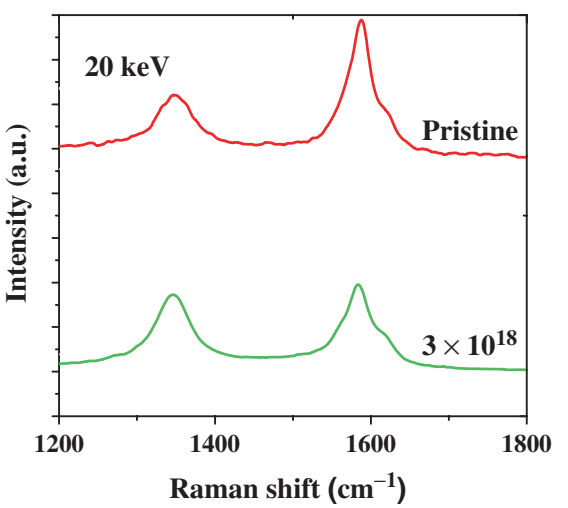

(b)

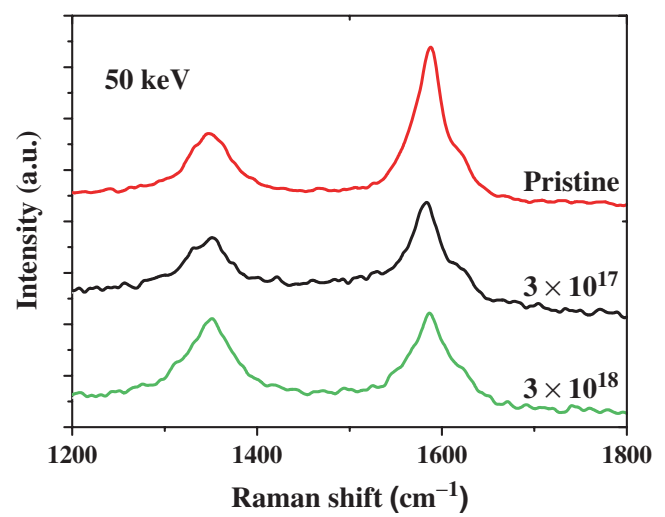

(c)

Figure 2. Raman spectra of CNTs irradiated with $\mathrm{Ar}^{+}$of different energies: (a) $3 \mathrm{keV}$, (b) $20 \mathrm{keV}$ (c) $50 \mathrm{keV}$ and different doses. 


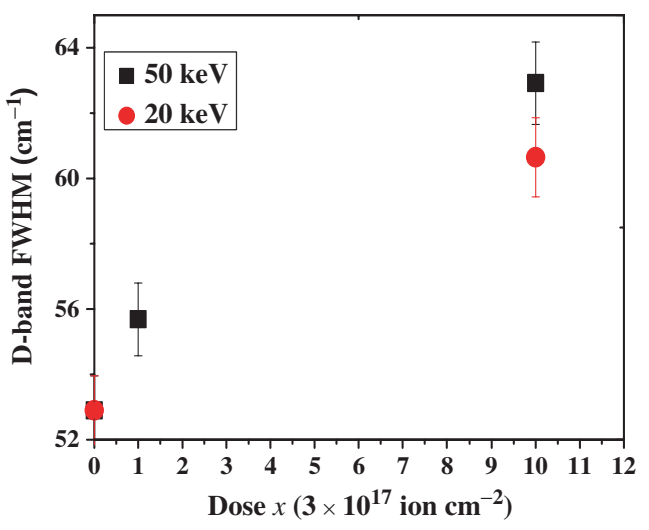

Figure 3. FWHM (D) of CNT samples irradiated with $\mathrm{Ar}^{+}$of different energies and doses.

evolution of the D and $\mathrm{G}$ bands which are characteristic of the presence of CNT, allows one to follow the structural evolution of CNTs with $\mathrm{Ar}^{+}$irradiation.

Figure 4 shows clearly that the relative intensity of the $\mathrm{D}$ to $\mathrm{G}$ bands is increased after $\mathrm{Ar}^{+}$irradiation, meaning that defects are reproduced on the CNTs. According to literature, ${ }^{9}$ ratio of $I_{\mathrm{D}} / I_{\mathrm{G}}$ is related to the coexistence of vacancies and induced transition of $\mathrm{sp}^{2} \rightarrow \mathrm{sp}^{3}$. The collision cascade of energetic ions to CNTs may create the structural defects especially via displacement of atoms and formation of vacancies due to kinetic energy transfer. High energetic ions could extremely damage the lattice and a great number of knock-on atoms will migrate as interstitials along the channels between walls of nanotubes leading to noteworthy high energy defects in CNTs that tend to react with each other. These complex reactions will weld the various CNTs and increase the $\mathrm{sp}^{3}$ density. It can also be deduced from this figure that the increment of $I_{\mathrm{D}} / I_{\mathrm{G}}$ is greater in low-energy bombardments. The reason for such behaviour is that at low energies the damage production grows with ion energy while at higher ion energies, although defect production in the CNTs drops as the nuclear collision cross-section $S\left(S=\pi P^{2}\right.$ where $P$ is the impact parameter) for defect production decreases, there are more energetic $\mathrm{C}$ primary recoils which damage the wall of CNTs. ${ }^{6,10}$ Therefore, it is suggested that at higher energies the decrease in damage due to the drop in cross-section and the damage enhancement due to the recoils approximately counterbalance each other. Delhaes $e t a l^{11}$ and Dresselhaus and Eklund ${ }^{12}$ suggested that due to equivalent behaviour of relative ratio of $I_{\mathrm{D}} / I_{\mathrm{G}}$ and $I_{\mathrm{D}^{\prime}} / I_{\mathrm{G}}$, the $\mathrm{D}^{\prime}$ band could be assigned to lattice defects which occurred inside the graphene atomic layer and induced a break in the two-dimensional translational symmetry. However there are little evidences on the dependence of the $D^{\prime}$ band intensity on lattice defects. Figure 5 shows the intensity ratio of $I_{\mathrm{D}^{\prime}} / I_{\mathrm{G}}$ as a function of irradiation dose which has shown very similar trend to $I_{\mathrm{D}} / I_{\mathrm{G}}$. These observations indicate that these two ratios can be considered as the most useful parameters for estimating the amounts of MWCNTs crystallinity.

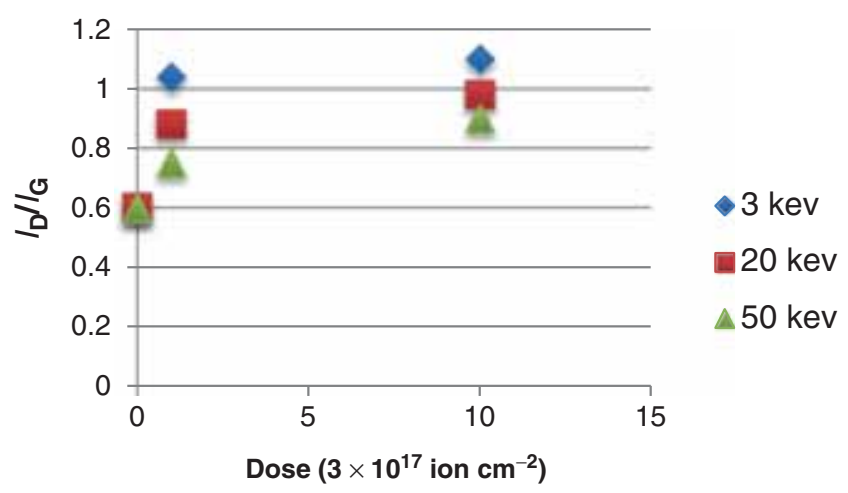

Figure 4. Intensity ratio of the $\mathrm{D}-\mathrm{G}$ bands $v s$. irradiation dose.

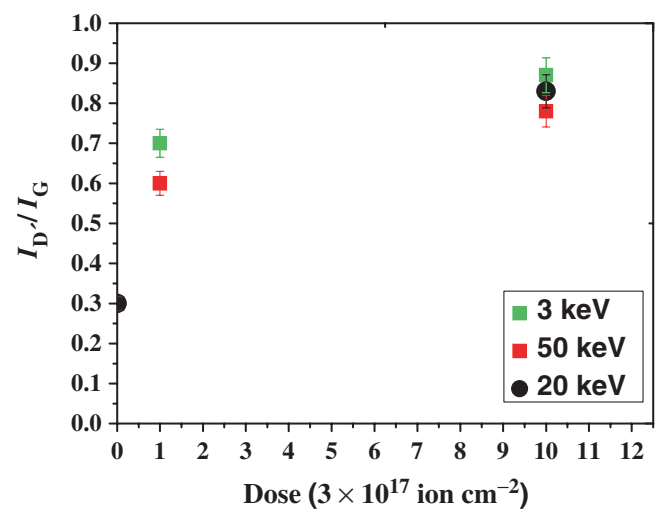

Figure 5. Intensity ratio of the $\mathrm{D}^{\prime}-\mathrm{G}$ bands $v s$. irradiation dose.

In order to make a precise comparison between the main features in the Raman spectra, the spectra were deconvoluted using the Gaussian function. The main advantage of this procedure is that one may clearly obtain the contribution of the peaks shoulders. Figure 6a-f shows the deconvoluted high-frequency spectra of $\mathrm{Ar}^{+}$-irradiated samples with different doses and energies. The deconvolution results of Raman spectra also show that the broad feature within 1200 $1800 \mathrm{~cm}^{-1}$ region is the envelop of the superposition of some important peaks and the $\mathrm{D}$ band. In addition the feature near $1580 \mathrm{~cm}^{-1}$ shifts to small frequencies. Thus, all vibration modes which are connected with the tangential atomic displacement of carbon are sensitive to the formation of radiation defects. This is in agreement with results of Ritter et al. ${ }^{13,14}$ Delhaes et $a l^{11}$ also indicated another first-order Raman band $\left(\mathrm{D}^{\prime}\right)$ appearing as a shoulder on the classical $\mathrm{G}$ band at approximately $1620 \mathrm{~cm}^{-1}$ which was induced by structural defects.

\subsection{SEM analysis}

Figure 7 illustrates SEM images of CNTs before and after bombardment with $50 \mathrm{keV}$ energy and $3 \times 10^{18}$ dose. As can 

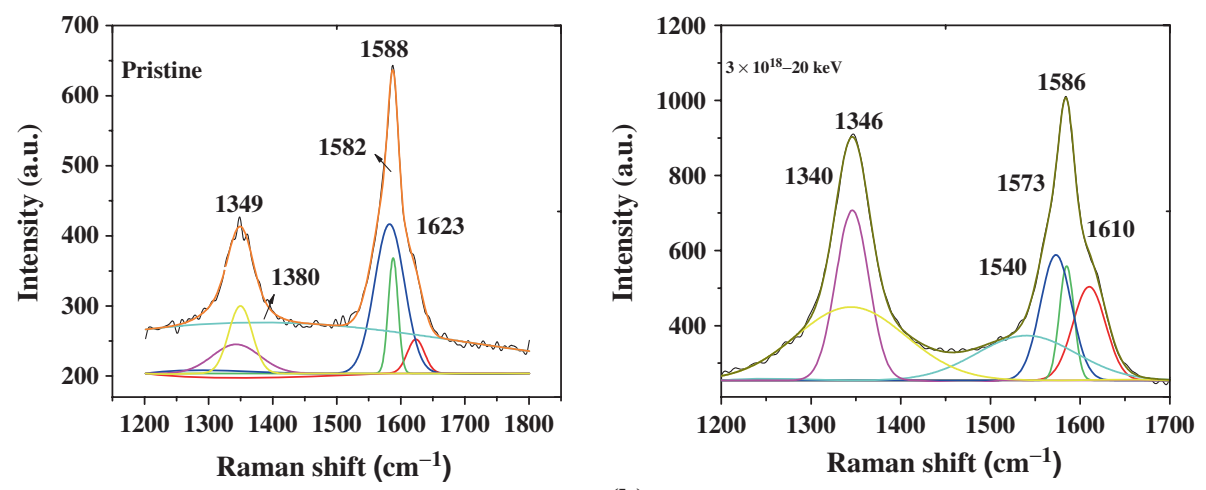

(a)

(b)

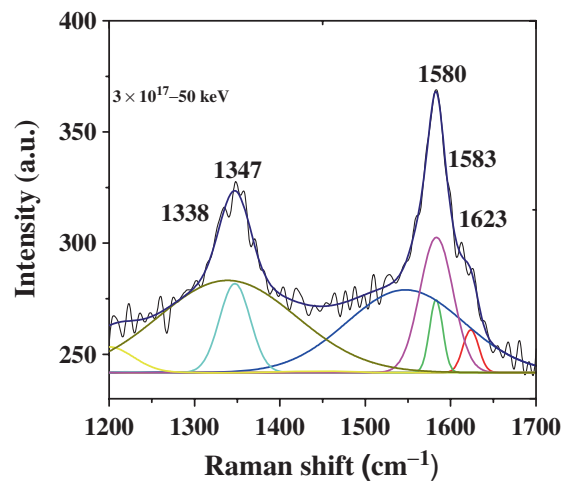

(c)
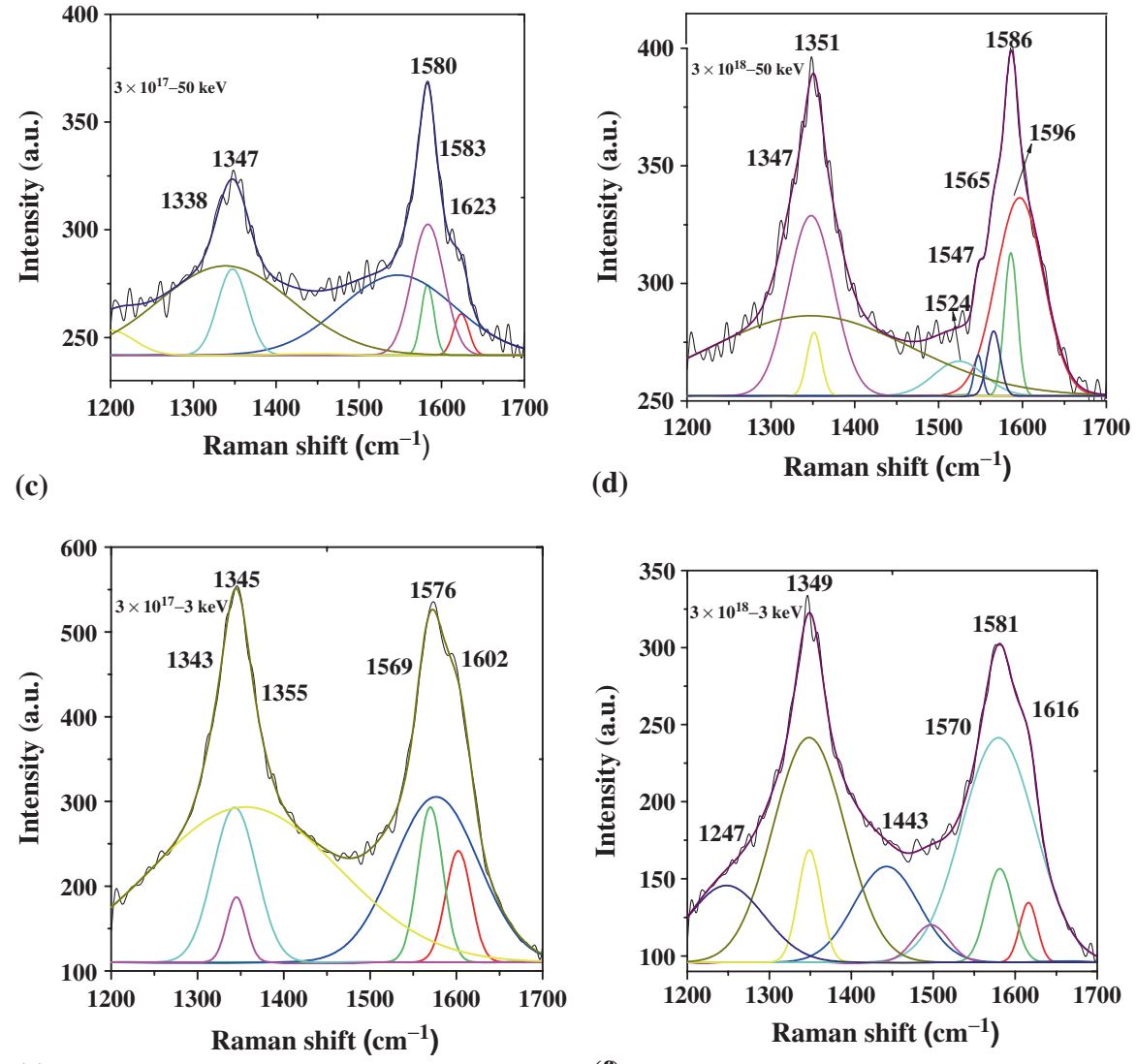

(e)

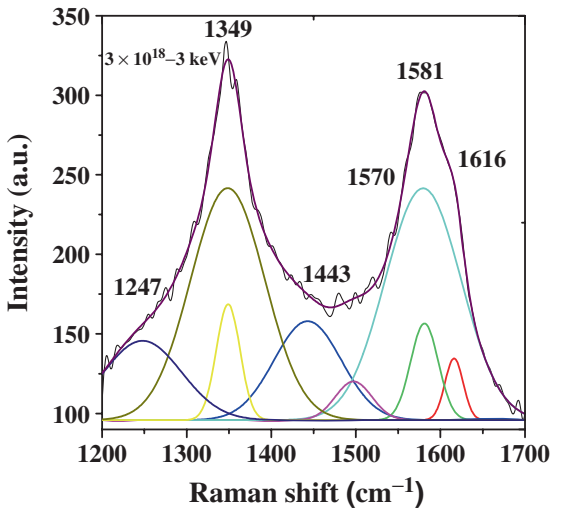

(f)

Figure 6. (a-f) Deconvoluted high-frequency spectra of $\mathrm{Ar}^{+}$-irradiated CNT samples with different doses and energies.

be seen, the CNTs are combined with each other after bombardment. This phenomenon results from the increase of the level of energy owing to creation of defects on the CNTs and increase in the mobility of atoms. The defects move towards each other and combine in order to decrease the level of energy.

\subsection{Sheet resistivity of samples}

Figure 8 presents a dose dependence of the sheet resistance of a MWCNT thin film. It can be observed that the sheet resistivity increases with radiation dose that due to defects created in CNTs. Defects are responsible for electron scattering and reduce the mean free path of the electrons, hence tend to decrease the conductivity. ${ }^{15,16}$

\section{Conclusion}

Radiation damage formation in CNTs is studied by Raman spectroscopy. Samples are irradiated with 3, 20 and $50 \mathrm{keV}$ energies of $\mathrm{Ar}^{+}$ions and $3 \times 10^{17}$ and $3 \times 10^{18}$ doses. The intensity ratio of the $\mathrm{D}-\mathrm{G}$ bands $\left(I_{\mathrm{D}} / I_{\mathrm{G}}\right)$ and FWHM 

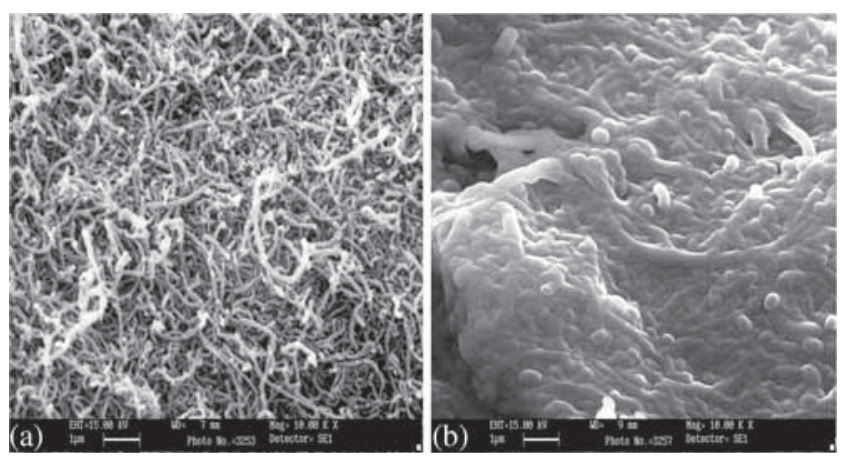

Figure 7. SEM images of CNTs (a) before and (b) after irradiation.

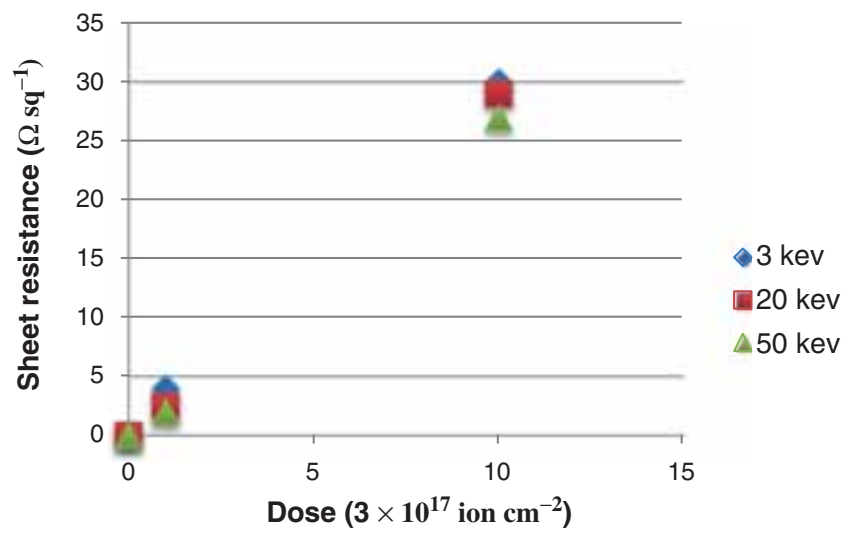

Figure 8. Sheet resistivity of CNTs vs. irradiation dose.

(D) increased with enhancement of irradiation dose. This ratio is relatively constant in 20 and $50 \mathrm{keV}$ energies. SEM images showed a post-irradiation aggregation of nanotubes. Sheet resistivity of CNTs are also increased as a function of irradiation dose.

\section{References}

1. Iijima S 1991 Nature 35456

2. Lehtinen O, Sun L, Nikitin T, Krasheninnikov A V, Khriachtchev L, Rodríguez-Manzo J A, Terronese M, Banhartb F and Keinonen J 2004 Nucl. Instrum. Methods: Phys. Res. 216 355

3. Chen C and Huang C 2008 Microporous Mesoporous Mater. 109549

4. Srivastava D, Donald W and David J 1995 Phys. Chem. B 103 4330

5. Basiuk V A and Basiuk E V 2007 Chemistry of carbon nanotubes (California: American Scientific Publishers) Chapter 1

6. Skakalova V, Maultezsch J, Osvath Z and Biro L P 2007 Phys. Status Solidi 138

7. Hong C, Lee J, Kalappa P and Advani S 2007 Compos. Sci. Technol. 671027

8. Pimenta M A, Marucci A, Empedocles S A, Bawendi M G, Hanlon E B, Dresselhaus G and Dresselhaus M S 1998 Phys. Rev. 5858

9. Compagnini G, Baratta G A, Cataliotti R S and Morresi A 2005 J. Raman Spectroscopy 26917

10. Pomoell J, Krasheninnikov A V, Nordlund K and Keinonen J 2003 Nucl. Instrum. Methods Phys. Res. 20618

11. Delhaes P, Couzi M, Trinquecoste M, Dentzer J and Hamidou H 2006 Carbon 443001

12. Dresselhaus M S and Eklund P C 2000 Adv. Phys. 49705

13. Ritter U, Scharff P, Siegmund C, Dmytrenko O P, Kulish N P, Prylutskyy Y I, Belyi N M, Gubanov V A and Komarova L I 2006 Carbon $\mathbf{4 4} 2694$

14. Ritter U, Scharff P, Dmytrenko O P, Kulish N P, Prylutskyy Y I, Belyi N M, Gubanov V A and Komarova L A 2007 Chem. Phys. Lett. 447252

15. Skakalova V, Woo Y S, Osvath Z and Biro L P 2006 Phys. Status Solidi 133346

16. Geng H Z, Lee D S, Kim K K, Kim J, Bae J J and Lee Y H 2008 Phys. Soc. 2979 